\title{
Poorly Cohesive Malignant Cellular Infiltrate
}

National Cancer Institute

\section{Source}

National Cancer Institute. Poorly Cohesive Malignant Cellular Infiltrate. NCI Thesaurus.

Code 195744.

A morphologic finding indicating the presence of a malignant cellular infiltrate

characterized by the presence of individual cells and/or tiny cellular aggregates. 